\title{
Abstract \\ Interplay of gouge, fluid pressure and porosity in fault zones
}

\author{
Kagan Tuncay $^{\mathrm{a}, *}$, Gonca Ozkan ${ }^{\mathrm{b}}$, Peter Ortoleva ${ }^{\mathrm{c}}$ \\ ${ }^{a}$ Earth Sciences, Utrecht University, 3508 TA Utrecht, The Netherlands \\ ${ }^{\mathrm{b}}$ Department of Mathematics, Bilkent University, Ankara, Turkey \\ ${ }^{\mathrm{c}}$ Department of Chemistry, Indiana University, Bloomington, IN 47405, USA
}

\begin{abstract}
The objective of this study is to demonstrate how fault dynamics may naturally be placed in the context of incremental stress theory, rock textural evolution modeling and standard conservation laws. Casting the fault dynamics problem in this framework naturally introduces rock memory for failure, fluid pressure effects and the autonomous nature of fault evolution. Poroelasticity, nonlinear viscosity and gouge are combined in an incremental stress rheology approach to examine the effect of changes in particle size distribution on fluid pressure in fault zones.
\end{abstract}

(c) 2003 Elsevier Science B.V. All rights reserved.

Keywords: Gouge; Faults; Rheology; Fluid pressure

\section{Introduction}

The complex nature of rock deformation is a result of many processes that are active in geologic environments. The list of processes includes poroelasticity, nonlinear viscosity, gouge, pressure solution and fracturing. Although some of these processes, such as poroelasticity, have been extensively studied separately, there is a very limited amount of work on the behavior of rocks when many of these processes simultaneously contribute to the evolution of stress and deformation. To gain insight into the nonlinear dynamics of stress and deformation and their evolution, a better understanding of the interaction of these deformation processes is necessary. For example, in

\footnotetext{
* Corresponding author. Department of Chemistry, Indiana University, Bloomington, IN 47405, USA. Tel.: +1-812-856-0981; fax: +1-812-855-8300.

E-mail address: ktuncay@indiana.edu (K. Tuncay).
}

the classical theory of poroelasticity, deviatoric stresses do not affect pore volume. Therefore, an approach considering classical poroelasticity alone can not explain fluid pressure changes as a result of changes in the deviatoric stress.

As geologic evidence is gathered on the involvement of fluids in faults, a growing number of researchers have started to study the effect of fluid pressure on fault mechanics. Rice (1992) and Byerlee (1990) suggested that relative weakness of some faults could be due to high intra-fault fluid pressure. Rice (1992) proposed that overpressuring could be maintained by the flow of deep fluids into the ductile roots of a fault zone. On the other hand, Byerlee's (1990) model explains high overpressure by conjecturing efficiently sealed compartments. Despite the growing interest in this research area, only a few quantitative models, limited to just a few processes, have been developed.

In an attempt at modeling fluid flow in partially sealed fault zones, Sleep and Blanpied (1994) as- 
sumed purely viscous rock behavior. The shear and bulk viscosities were assumed to be functions of the pore and fracture volume fractions which were taken to evolve via two empirical ordinary differential equations. The pressure equation was based on the conservation of fluid phase and included both increase in pressure because of compaction and decrease in pressure due to escape of fluid. In the case of a single fault system, Sleep and Blanpied (1994) showed that the increased fluid pressure allows frictional failure in earthquakes at shear tractions far below those required when fluid pressure is hydrostatic. However, in their numerical treatment, an predetermined shear traction drop was imposed (a fraction of its value) and the displacement, pore volume and fluid pressure were reset. In other words, in the case of a single fault system, an oscillation was imposed, not simulated. Segall and Rice (1995) modified the rate- and statedependent friction model to include fluid pressure and porosity (based on porosity changes observed in gouge experiments with overcompacted rocks). Their final set of equations includes an ordinary differential equation for velocity dependent porosity based on experimental observations. In gouge experiments (for overcompacted rocks), if the normal stress is kept constant, an increase in porosity is observed. The increase in pore volume results in a decrease in fluid pressure and an increase in normal stress which, in turn, tends to stabilize faulting.

Although grain size distributions of natural fault gouge have been studied extensively (Morrow and Byerlee, 1989; Marone and Scholz, 1989; Ozkan and Ortoleva, 2000), to our best knowledge, quantitative modeling of gouge and its effect on fluid pressure in shear zones is not extensively studied. Recently, Ozkan and Ortoleva (2000) studied the evolution of grain size distribution with a Markov model and confirmed their model with experimental data of Morrow and Byerlee (1989).

In this short paper, we present a mathematical model that allows one to couple poroelasticity, viscosity and gouge to fluid flow in a self-consistent manner.

\section{Model development}

We suggest that rigorous models of rock behavior should be of the Markov type-i.e., the rate of change of rock state should only depend on the instantaneous rock state and not on prior history. Stress and strain are related, through rock rheology, to rock texture $\Theta$ (a set of variables including grain size, shape, packing and mineralogy). Pressure solution and grain breakage imply that the rate of change of $\Theta$ depends on stress, denoted by $\sigma$. If $\Theta$ satisfies

$\frac{\mathrm{d} \Theta}{\mathrm{d} t}=G(\Theta, \sigma)$

then, in principle, $\Theta(t)$ is a functional of $\sigma$, i.e. depends on $\sigma\left(t^{\prime}\right)$ for all $t^{\prime}<t$, i.e. on the stress history: $\Theta=\Theta[\sigma]$. As rheology depends on $\Theta$, we see that $\Theta[\sigma]$ reflects the entire prior stress history and not just the instantaneous value of stress. Clearly, however, this "memory" in a theory wherein $\Theta$ is not coevolved with $\sigma$ is an artifact of the incompleteness of a rock deformation model that attempts to avoid coevolving $\Theta$ with stress. While there are many stress-strain histories that could lead to the instantaneous state of a rock, only the latter is key to predicting its failure and other behavior.

To predict faulting and other rock failure phenomena, we introduce a model that accounts for rock competency. Let "rock competency" measure the fraction of grain surface that is attached to other grains. Thus, $\Gamma$ is in the range $0<\Gamma<1$. Large $\Gamma$ implies competency, while in a low $\Gamma$ rock, there are few intact grain-grain contacts. Thus, rheologic quantities such as rock strength or viscosity are strongly dependent on $\Gamma$. Schematically, our model is as follows. The equation of motion of $\Gamma$ is taken in the form

$\frac{\mathrm{D} \Gamma}{\mathrm{D} t}=R(\Gamma, F)$

where $F$ is a failure function that depends on macroscopic stress, fluid pressure, rock texture, mineralogy and temperature. In three dimensions, the failure function can be assumed to take the form proposed by Drucker and Prager: $F=a J_{1}+J_{2}^{1 / 2}-b$, where $J_{1}$ is the first invariant of the effective stress tensor and $J_{2}$ is the second invariant of the deviatoric effective stress tensor. The coefficients $a$ and $b$ can be expressed in terms of angle of internal friction and cohesion determined from conventional triaxial compression experiments. If the dynamics of $\Gamma$ is rela- 
tively fast, its evolution is closely related to the shape of the curve $R=0$. The $\Gamma$ dynamics is, in a sense, a cooperative phenomenon, i.e., a decrease in competence fosters more rapid $\Gamma$ decline. This is captured by the qualitative picture of Fig. 1, where a schematic evolution path in the $F, \Gamma$ plane is shown (see Tuncay et al., 2001 for a detailed discussion of failure/healing dynamics).

The integration of deformation mechanisms can most effectively be done using an incremental stress approach. In this theory, the rate of strain is written as a sum of terms, each accounting for a particular process $\left(j=1,2, \ldots N_{\mathrm{d}}\right)$ for a system with $N_{\mathrm{d}}$ deformation processes (Tuncay et al., 2000a):

$\underline{\underline{\dot{\varepsilon}}}=\sum_{j=1}^{N_{\mathrm{d}}} \underline{\dot{\varepsilon}}^{j}$

Some of the most important deformation mechanisms are poroelasticity, nonlinear viscosity, gouge, fracturing and pressure solution (Tuncay et al., 2000a,b; Tuncay and Ortoleva, 2001).

The rate of strain due to poroelasticity is given by

$\underline{\underline{\dot{\varepsilon}}}^{\text {elasticity }}=\underline{\underline{\underline{C}}}^{-1}(\Theta) \frac{\mathrm{D}}{\mathrm{D} t}(\underline{\underline{\sigma}}+\alpha(\Theta) p \underline{\underline{I}})$

for fourth-rank matrix of poroelastic coefficients $\underline{C}$ and effective stress coefficient $\alpha ; \mathrm{D} / \mathrm{D} t$ represents $\underline{\overline{\bar{a}}}$

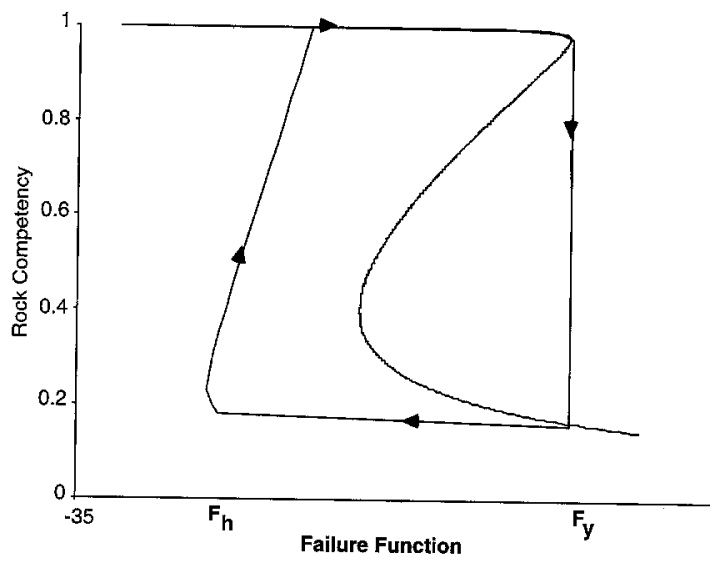

Fig. 1. Schematic competence $(I)$ and failure function $(F)$ plane illustrating the cooperative aspects of rock failure. When $\Gamma$ is near unity, the rock is competent, but when $F$. exceeds $F_{\text {yield, it is }}$ compromised. However, for $F<F_{\mathrm{h}}$, competence is regained through chemical healing processes. If a point is on the right of the $\mathrm{S}$-shaped curve, rock competency decreases; otherwise, it increases. material time derivative measuring the rate of change of a tensor in time with respect to a local reference frame fixed to a translating, rotating material volume element. The bulk and shear moduli of the drained porous medium, and the effective stress coefficient of the medium, can be computed in terms of the rock texture and mineral elastic properties using Berryman's $(1980,1986)$ approach.

The inelastic mechanical contribution to the rate of strain is cast in the present approach as a nonlinear viscosity law in the form

$\underline{\underline{\varepsilon}}^{\text {viscosity }}=\underline{\underline{\underline{\eta}}}^{-1}(\underline{\underline{\sigma}}+\tilde{\alpha} p \underline{\underline{I}})$

The fourth-rank viscosity tensor $\underline{\underline{\underline{\eta}}}$ depends on stress, fluid pressure and texture. The $\stackrel{\equiv}{\bar{E}}$ second term in the effective stress involves a coefficient $\tilde{\alpha}$ that is usually taken in the literature to be unity. Viscosities are strong functions of rock competency and grain size (Tuncay et al., 2000a).

Ozkan and Ortoleva (2000) presented a model to calculate the grain size distribution and average grain size evolution as a function of induced shear strain. A Markov model was used to evolve the particle size distribution. A breaking probability was introduced that accounts for the observed grinding limit and the development of a bimodal distribution that depends on the degree of shearing and the normal effective stress. The model was calibrated with particle size distribution measurements on Ottowa sand (Morrow and Byerlee, 1989). To illustrate the gouge dynamics modeling, consider a monomineralic system. Let $\xi(n$, $t)$ be the number per rock volume of grains with $n$ unit cells of the crystalline solid. Thus, $\xi(n, t)$ is the particle size distribution. If $\xi$ is the column vector of $\xi$ values $\left(\underline{\xi}=\xi(1, t), \xi(2, t), \ldots \xi\left(10^{23}, t\right) \ldots\right)$, then $\xi$ satisfies

$\frac{\mathrm{D} \underline{\underline{\xi}}}{\mathrm{D} t}=\underline{\underline{M}} \underline{\xi}$

where $\underline{M}$ is the transition probability for the assumed Markov process. The $\underline{\underline{M}}$ matrix depends on stress, fluid pressure and temperature and, in addition, on the composition of the pore fluid due to diagenesis. Thus, grains are broken due to gouge but grow/ dissolve due to fluid rock reactions. The model is based on the binary breaking of individual grains 
into fragments of arbitrary size. The breakup probability incorporates the observed effects of shear strain, confining stress and fluid pressure. We refer to Ozkan and Ortoleva (2000) for the details of the mathematical model. The feedback between gouge formation and deformation is completed by the rate of strain due to gouge. In general, the rate of strain due to gouge formation is a function of pressure, stress tensor, texture, and history of shear strain. For loosely lithified sediments, it can be assumed that there exists a relationship between porosity and sorting based on the experimental results of Beard and Weyl (1973). Then the rate of strain due to gouge formation is obtained as

$\underline{\underline{\dot{\varepsilon}}}^{\text {gouge }}=\frac{1}{1-\phi} \frac{\mathrm{D} \phi}{\mathrm{D} s} \frac{\mathrm{D} s}{\mathrm{D} t}$

where $\phi$ and $s$ are the porosity and sorting parameter, respectively.

The force balance equation completes the set of equations for stress and deformation

$\sum_{i^{\prime}=1}^{3} \frac{\partial \sigma_{i i^{\prime}}}{\partial x_{i^{\prime}}}+f_{i}=0$

for body force $f_{i}$ which, for gravity, is given by

$f_{i}=g \rho_{\mathrm{m}} \delta_{i 3}$.

The mass balance equation of fluid phase is given by

$\frac{\partial \phi \rho_{\mathrm{f}}}{\partial t}+\underline{\nabla} \cdot\left(\underline{v}_{\mathrm{f}} \phi \rho_{\mathrm{f}}\right)=0$

Using a linear state equation in the form of $\rho_{\mathrm{f}}=\bar{\rho}_{\mathrm{f}}\left(1+\beta\left(p-p_{0}\right)\right)$ with compressibility $\beta$, Darcy law relative to moving solids and mass balance equation of solid phase, the fluid flow equation is obtained as

$\phi \bar{\rho}_{\mathrm{f}} \beta \frac{\mathrm{D} p}{\mathrm{D} t}=\underline{\nabla} \cdot \frac{k \rho_{\mathrm{f}}}{\mu_{\mathrm{f}}}\left(\underline{\nabla} p+\rho_{\mathrm{f}} g \underline{z}\right)-\rho_{\mathrm{f}} \underline{\nabla} \cdot \underline{u}$

where $k$ is the permeability that depends on the grain size distribution and porosity. Once the solid velocity field is obtained, porosity is solved from the mass balance of solid phase under the assumption of incompressible solid grains

$$
\begin{aligned}
\frac{\mathrm{D} \phi}{\mathrm{D} t} & =(1-\phi) \underline{\nabla} \cdot \underline{\underline{u}} \\
& =(1-\phi) \operatorname{tr}\left(\underline{\underline{\dot{\varepsilon}}}^{\text {elasticity }}+\underline{\underline{\dot{\varepsilon}}}^{\text {viscosity }}+\underline{\underline{\dot{\varepsilon}}}^{\text {gouge }}\right)
\end{aligned}
$$

which shows the dependence of porosity evolution on the individual rate of strain terms.

\section{Conclusion}

Fault mechanics modeling must be carried out in three spatial dimensions and via a model that incorporates a full suite of crustal reaction, transport, and mechanical processes. We believe that the present mathematical model, as applied to competency, fluid flow, diagenesis and deformation in each macrovolume element of a finite element approach, will allow for first principles fault simulation. As such a model accounts for the full range of rock states (intact to failed), a three-dimensional finite element approach will allow for the prediction of fault morphology (thickness, overall shape, branching) as well as reactivation of healed sections of a previously active zone.

\section{References}

Beard, D.C., Weyl, P.K., 1973. Influence of texture on porosity and permeability of unconsolidated sand. AAPG Bulletin 57, $349-369$.

Berryman, J.G., 1980. Long-wavelength propagation in composite elastic media: I. Spherical inclusions. Journal of the Acoustical Society of America 68, 1809-1819.

Berryman, J.G., 1986. Effective medium approximation for elastic constants of porous solids with microscopic heterogeneity. Journal of Applied Physics 59, 1136-1140.

Byerlee, J.D., 1990. Friction, overpressure and fault normal compression. Geophysical Research Letters 17, 2109-2112.

Marone, C., Scholz, C.H., 1989. Particle size distribution and microstructures within simulated fault gouge. Journal of Structural Geology 11, 799-814.

Morrow, C.A., Byerlee, J.D., 1989. Experimental studies of compaction and dilatancy during frictional sliding on faults containing gouge. Journal of Structural Geology 11, 815-825.

Ozkan, G., Ortoleva, P., 2000. Evolution of gouge grain size distribution: a Markov model. Pure and Applied Geophysics 157 (3), 449-468. 
Rice, J.R,, 1992. Fault stress states, pore pressure distributions, and the weakness of the San Andreas Fault. In: Evans, B., Wong, T.-F. (Eds.), Fault Mechanics and Transport Properties in Rocks. Academic Press, London, pp. 475-503.

Segall, P., Rice, J.R., 1995. Dilatancy, compaction, and slip instability of a fluid-infiltrated fault. Journal of Geophysical Research 100, 22155-22171.

Sleep, N.H., Blanpied, M.L., 1994. Ductile creep and compaction: a mechanism for transiently increasing fluid pressure in mostly sealed fault zones. Pure and Applied Geophysics 143, 9-40.

Tuncay, K., Ortoleva, P., 2001. Salt tectonics as a self-organizing process: a reaction, transport and mechanics model. Journal of Geophysical Research 106, 803-818.

Tuncay, K., Park, A., Ortoleva, P., 2000a. Sedimentary basin deformation: an incremental stress rheology approach. Tectonophysics 323, 77-104.

Tuncay, K., Park, A., Ortoleva, P., 2000b. A forward model of three dimensional fracture orientation and characteristics. Journal of Geophysical Research 105, 16719-16735.

Tuncay, K., Khalil, A., Ortoleva, P., 2001. Failure, memory and cyclic fault movement. Bulletin of Seismological Society of America 91, 538-552. 